\title{
Comportamiento agroindustrial de seis variedades de Caña de azúcar (Saccharum spp.) para Panela en Barbosa (Colombia)
}

\author{
Agroindustrial performance of sugarcane (Saccharum spp.) \\ varieties for panela in Barbosa (Colombia).
}

\author{
Ayda Fernanda Barona Rodríguez ${ }^{1 *}$, Eduar Yovany Antolinez Sandoval ${ }^{2}$, Juan Gonzalo López Lopera ${ }^{3}$, \\ Carlos Arturo Viveros Valens ${ }^{4}$, Javier Jiménez Vargas ${ }^{5}$, Juan Carlos Ángel Sánchez ${ }^{6}$, \\ Andrea Lizeth Ballesteros Araque ${ }^{7}$, Germán Andrés Vargas Orozco ${ }^{8}$
}

DOI: https://doi.org/10.19053/01228420.v18.n3.2021.12856

RESUMEN: En Colombia, la producción de caña de azúcar es el segundo renglón más importante en la producción agrícola después del café, $55 \%$ del total del área cultivada se dedica a la producción de panela (Azúcar no centrifugado-NCS, por sus siglas en inglés) labor que se realiza en 29 de los 32 departamentos del país. Los bajos rendimientos y la pérdida de calidad genética de los materiales que actualmente se cultivan ha traído consigo la necesidad de evaluar nuevas variedades de caña de azúcar que se adapten a diversos ambientes y que cuenten con una alta producción de panela. Teniendo en cuenta lo anterior, entre noviembre de 2018 y mayo de 2020, por parte de la Corporación Colombiana de Investigación Agropecuaria - Agrosavia, bajo las condiciones agroecológicas de Barbosa (Santander, Colombia), se evaluó el comportamiento agroindustrial de seis variedades de caña de azúcar para producción de panela (CC 11600, CC 10-450, CC 99-2461, CC 00-3257, CC 91-1606 y CC 00-3771) provenientes del centro de investigación de la caña de azúcar de Colombia - Cenicaña; se estableció un diseño de bloques completos al azar con cuatro repeticiones para el comportamiento agronómico y tres repeticiones para la evaluación industrial. En lo agronómico, los materiales genéticos presentaron promedios de $2,81 \mathrm{~m}$ para altura de tallo a punto de quiebre, $2,8 \mathrm{~cm}$ de diámetro de tallo, porcentaje de intensidad de infestación de Diatraea del $10,66 \%$ y 118,92 toneladas de caña por hectárea; para las variables industriales, sen encontraron valores promedio de $21,06^{\circ}$ Brix en jugo y 12,78 toneladas de panela por hectárea, las variables evaluadas referentes a calidad de panela se ajustaron a las requeridas en las NTC 1311:2009 y Resolución 000779 de 2006 del Ministerio de Protección Social. Las variedades CC 11-600 y CC 00-3267 presentaron el mejor comportamiento agroindustrial en el primer ciclo productivo.

PALABRAS CLAVE: Calidad de los alimentos, Edulcorantes, Panela, Productos Vegetales Procesados, Variedades de alto rendimiento.
ABSTRACT: In Colombia, sugarcane production is the second most important line of agricultural production after coffee, $55 \%$ of the total cultivated area is dedicated to the production of panela (non-centrifuged sugar - NCS), a task that is carried out in 29 of the 32 departments of the country. Low yields and the loss of genetic quality of the materials currently being cultivated have brought about the need to evaluate new varieties of sugarcane that adapt to different environments and have a high production of panela. Considering the above, between November 2018 and May 2020, by the Colombian Agricultural Research Corporation - Agrosavia, under the agroecological conditions of Barbosa (Santander, Colombia), the agroindustrial behavior of six sugarcane varieties for panela production (CC 11-600, CC 10-450, CC 99-2461, CC 00-3257, CC 91-1606 and CC 00-3771) from the Colombian sugarcane research center - Cenicaña were evaluated; A randomized complete block design was established with four repetitions for agronomic performance and three replicates for industrial evaluation. Agronomically, the genetic materials presented averages of $2.81 \mathrm{~m}$ to plant height, $2.8 \mathrm{~cm}$ stem diameter, percentage of Diatraea infestation intensity of $10.66 \%$ and 118.92 tons of cane per hectare; for the industrial variables, average values of $21.06^{\circ}$ Brix in juice and 12.78 tons of panela per hectare were found; the evaluated variables referring to panela quality were adjusted to those required in the NTC 1311: 2009 and Resolution 000779 of 2006 of the Ministry of Social Protection. Varieties CC 11-600 and CC 003267 showed the best agroindustrial performance in the first production cycle.

KEY WORDS: Food quality, Sweeteners, Panela, Processed Vegetable Products, High Yielding Varieties.

\section{FECHA DE RADICACIÓN: 10 de julio de 2021 FECHA DE APROBACIÓN: 23 de agosto de 2021}

CitAR Como: Barona Rodríguez, A. F., Antolinez Sandoval, E. Y., López Lopera, J. G. ., Viveros Valens, C. A. ., Jiménez Vargas, J. ., Ángel Sánchez, J. C. ., Ballesteros Araque, . A. L. ., \& Vargas Orozco, G. A. . Comportamiento agroindustrial de seis variedades de Caña de azúcar (Saccharum spp.) para Panela en Barbosa (Colombia). Cien. Agri., 18(3): 15-28. https://doi.org/10.19053/01228420.v18.n3.2021.12856

1, 2, 5, 7 Corporación Colombiana de Investigación Agropecuaria - Agrosavia. Centro de Investigación Tibaitatá, Sede Cimpa (Barbosa, Colombia). ${ }^{1}$ https://orcid.org/0000-0001-8487-2727; (D) ${ }^{2}$ https://orcid.org/0000-0003-0145-6002 (D) ${ }^{5}$ https://orcid.org/0000-0003-2563-7473

${ }^{7}$ https://orcid.org/0000-0001-5880-6347

3 Corporación Colombiana de Investigación Agropecuaria - Agrosavia. Centro de Investigación El Nus (San Roque, Colombia) (D) ${ }^{3}$ https://orcid.org/0000-0001-5416-8229

4, 6, 8 Centro de Investigación de la Caña de Azúcar de Colombia - Cenicaña. (Cali, Colombia). (D) 4 https://orcid.org/0000-0001-6824-1722 (D) ${ }^{6}$ https://orcid.org/0000-0003-1536-9938 (D) ${ }^{8}$ https://orcid.org/0000-0002-3232-8356

Autor de correspondencia: abarona@agrosavia.co 


\section{Introducción}

El azúcar no centrifugado (NCS, por sus siglas en inglés), es la denominación técnica usada por la Organización de las naciones unidas para la alimentación y la agricultura (FAO) para un producto sólido no refinado que se obtiene por evaporación del jugo de la caña de azúcar (FAO, 1994); recibe diferentes nombres alrededor del mundo como azúcar muscovado (Filipinas y algunos países africanos), jaggery (Uganda, Kenia e India), gur (India), khandsari (Asía meridional), papelón (Venezuela y países de África Central), piloncillo (México), rapadura (Argentina, Brasil y Cuba), tapa dulce (Costa Rica), panela (Colombia, Guatemala, Bolivia, Ecuador, Honduras, Nicaragua y Panamá) atado de dulce (Nicaragua), chancaca (Perú y Chile) y empalizado (Bolivia) (Flórez-Martínez et al., 2021; Velásquez et al., 2019).

De acuerdo con Garcia et al. (2017), la producción mundial de panela (NCS) se encuentra alrededor de 10 millones de toneladas por año, siendo los principales productores India ( 50\%), Colombia ( 17\%) y Pakistán ( 10\%).

La panela está compuesta principalmente por sacarosa (65-85\%), azucares reductores (10-15\%), agua (3-10\%) y sustancias no solubles como grasa, proteinas, minerales, hierro y fosforo que normalmente se encuentran $<1 \%$ (Velásquez et al., 2019; Jaffé, 2015).

En Colombia, dentro del grupo de los perennes, la caña de azúcar ocupa el segundo lugar en área sembrada después del café (Ramírez-Durán et al., 2014), su cultivo está destinado principalmente a la producción de azucar o etanol (45\%) y panela (55\%) (Murcia-Pardo \& Ramírez-Durán, 2017). Para el año 2019, la producción panelera contó con un área sembrada de 200.470 ha, y una producción de 1.098.207 t, siendo los principales productores Santander, Cundinamarca, Antioquia y Boyacá con cerca del 56\% de la panela del país; el rendimiento calculado fue de 6,21 t ha-1 (Rodríguez et al., 2019).

La investigación sobre la panela ha ido evolucionando a partir de la mejora de las variedades de caña de azúcar y la evaluación de su proceso de eficiencia energética (Flórez-Martínez, Contreras-Pedraza, \& Rodríguez, 2021).

La necesidad de proporcionar una variedad de caña de azucar que responda favorablemente a las condiciones ambientales, resisitente a plagas y enfermedades, y con altos rendimientos, ha motivado a las organizaciónes publicas y privadas de diferentes paises productores a la busqueda de nuevas variedades con base a cruzamientos, que se han evaluado con las variedades comerciales tradicionales (Duarte Álvarez, 2019). La caña de azúcar es un producto principal en los más de 130 paises y territorios donde se cultiva, ocupando alrededor de $1,9 \%$ de la superficie total cultivada a nivel mundial (Gómez-Merino et al, 2017) 
La relación entre el genotipo y la calidad de la panela ha sido estudiada desde hace varios años, esto ha llevado a la obtención de cultivares de caña de azúcar para producción de panela por parte de institutos de investigación agricola en paises como Colombia y Venezuela (Jaffé, 2015).

Las interacciones que se dan entre los cultivares y el medio ambiente $\left(\mathrm{G}^{*} \mathrm{~A}\right)$ son un factor determinante en la producción y la maduración de la caña de azúcar, es por tanto importante estudiar estas relaciones para identificar los ambientes en los cuales los materiales geneticos expresen de una mejor manera todo su potencial (Gimenes-Catelan et al., 2022); se deben hacer validaciones de ensayos en espacio (localidades), y tiempo (años y cepas) para de esta forma aprovechar de la mejor manera la interacción G*A (Suárez et al., 2019).

La investigación de muchos paises que han trabajado en el mejoramiento genetico de la caña de azúcar se han centrado en seleccionar variedades con altos contenidos de azúcar y que tengan una maduración precoz (Mendoza-Batista et al., 2019) excelente rendimiento agrícola y industrial, con calidad de jugos y con grados de resistencia a plagas y enfermedades (Delgado-Mora et al., 2016).

Una de las principales líneas de investigación del sistema productivo de la caña de azúcar para la producción de panela está centrada en poder identificar variedades que se destaquen por una alta producción de caña y rendimiento de panela por hectárea, y que se adapten a las condiciones particulares de las zonas paneleras (Lopez \& Tamayo, 2017; Vera-Gutiérrez et al., 2019). En Colombia, estas variedades son desarrolladas por el Centro de Investigación de la Caña de Azúcar de Colombia - Cenicaña, con el propósito de mejorar la producción de caña y azúcar (Lopez \& Tamayo, 2017), estas variedades son evaluadas con enfoque de producción panelera por instituciones como la Corporación Colombiana de Investigación Agropecuaria - Agrosavia (Ramírez-Durán et al., 2014).

El objetivo de la presente investigación fue observar el comportamiento agroindustrial de seis variedades de caña de azúcar provenientes de Cenicaña y evaluadas con fines de producción de panela bajo las condiciones de Barbosa, Santander, Colombia.

\section{Materiales y Métodos}

\section{A. Ubicación}

La evaluación de las variedades tuvo una duración de 17 meses comprendidos entre noviembre de 2018 y mayo de 2020. Se estableció en el C.I Tibaitatá sede Cimpa de la Corporación Colombiana de Investigación Agropecuaria 
- Agrosavia ubicada en el municipio de Barbosa (Santander), con coordenadas $5,947674^{\circ} \mathrm{N}$ y $73.606870 \mathrm{~W}, 1621 \mathrm{msnm}$, temperatura media de $22^{\circ} \mathrm{C}$ y precipitación de $1800 \mathrm{~mm}$ año-1 (Información estación meteorológica Agrosavia Cimpa).

\section{B. Material vegetal}

Se evaluaron las siguientes seis variedades de caña de azúcar: Cenicaña Colombia (CC) 11-600, CC 10-450, CC 99-2461, CC 00-3257, CC 91-1606 y CC 00-3771. Dichas variedades fueron introducidas a la región por su alto potencial agroindustrial y comportamiento fitosanitario en el Valle del Cauca, especialmente a enfermedades de importancia económica para el cultivo como roya naranja, roya café y virus de la hoja amarilla.

\section{Diseño experimental}

El diseño experimental empleado fue de bloques completos al azar BCA, con seis tratamientos (variedades) y cuatro repeticiones, para un total de 24 unidades experimentales, se optó por este diseño para eliminar la posible interferencia de la pendiente del lote donde se estableció el ensayo. Cada unidad experimental estuvo conformada por cuatro surcos de $5 \mathrm{~m}$ de longitud separados entre sí a $1.30 \mathrm{~m}$. La parcela fue establecida con plantas pregerminadas de caña de azúcar, garantizando pureza varietal y sanidad vegetal (Ramírez-Durán et al., 2019). Para la siembra se realizó ahoyado a 10 $\mathrm{cm}$ de profundidad con herramienta manual a una distancia de 0,60 m entre plantas y $1,30 \mathrm{~m}$ entre surcos.

El control de arvenses se realizó a los 2 y 4 meses posterior a la siembra para evitar la competencia durante el periodo de rápido crecimiento. La fertilización se realizó a los 15 días después de cada control de arvenses empleando una mezcla química comercial 17-6-18-2 (Mg), el fertilizante fue cubierto con suelo y hojarasca del cultivo para minimizar las perdidas por volatilización.

\section{Cosecha}

La cosecha de las variedades se realizó a los 17 meses después de la siembra teniendo en cuenta el índice de madurez (IM) presentado. El IM es un indicativo del grado de maduración de la caña determinado por la relación del Brix Apical y el Brix basal, Ramírez et al. (2014a) manifiestan que se considera que la caña alcanzó su madurez cuando el IM se encuentra entre 0,95-1.

Al momento de la cosecha se seleccionaron al azar 10 tallos por cada unidad experimental y se evaluaron las siguientes variables: 1) altura de tallo a punto de quiebre - $m(A L P Q)$, cuantificada desde la base del tallo hasta la primera 
lígula o cuello visible utilizando una mira topográfica de $5 \mathrm{~m}$ de largo. 2) diámetro del tallo - cm (DIATA) mediante un calibrador digital marca mitutoyo (precisión 0,01 mm), registrado de la parte media del tallo. 3) índice de madurez (IM), dado por la relación entre el brix apical (tomado en el séptimo u octavo entrenudo contado desde la primera lígula visible) y el brix basal (tomado en el tercer o cuarto entrenudo contado desde la base del suelo), para tal fin se tomó muestra del jugo del tallo con un punzón y se empleó un refractómetro digital de mano Hanna H196801 de 0-85 \%Brix; porcentaje de intensidad de infestación de Diatraea spp. (\%.l) determinado de acuerdo con la metodología desarrollada por Cenicaña (2005).

Para la obtención de la población de tallos por metro lineal, se realizó el conteo de todos los tallos presentes en la unidad de muestreo y estos fueron pesados para estimar las toneladas de caña obtenidas por hectárea (TCH).

\section{E. Análisis de jugos de caña y panela en laboratorio}

En la planta piloto de la sede Cimpa, se realizó el procesamiento de tallos para la obtención de panela, la extracción de jugos se realizó en un molino de tres masas marca "El panelero". Al jugo obtenido se le realizó medición a nivel de laboratorio de variables de calidad como la determinación de los sólidos solubles totales ('Brix) mediante un refractómetro digital marca Anton Paar con corrección de temperatura a $20^{\circ} \mathrm{C}, \mathrm{pH}$, mediante el potenciómetro seven easy (Mettler Toledo), azúcares reductores (\%), mediante el método volumétrico Eynon Lane, sacarosa (\%) mediante polarimetría con uso de polarímetro (ATACO SAC-i) y pureza de sacarosa entre la relación de sacarosa y los sólidos solubles totales.

Posteriormente, los jugos fueron procesados hasta la obtención de panela y se le realizaron los mismos análisis realizados al jugo, se determinó humedad, por método gravímetro con secado es estufa marca Memmert U30. Para la evaluación tanto en jugo como en panela se tuvo en cuenta la metodología descrita por Ramírez-Durán et al. (2014b), para la caracterización química de la panela y los valores requeridos se tuvo en consideración los consignados en la NTC 1311:2009 y en la resolución 779 de 2006 del Ministerio de Salud y Protección Social para los diferentes requisitos de calidad. Adicionalmente, se determinaron variables de tipo industrial como: 1) Porcentaje de conversión a panela (CP): determinada por el peso de la panela obtenida en relación con el peso de los tallos molidos y la extracción obtenida de jugo; 2) toneladas de panela por hectárea (TPH): teniendo en cuenta las toneladas de caña por hectárea (TCH) y el porcentaje de conversión a panela (CP) y 3) Contenido de cachaza (Chz): dado por el peso total de las impurezas extraídas en el proceso de la producción de la panela en relación con el peso total de los tallos procesados para la muestra de panela. 


\section{F. Análisis de resultados}

Los resultados, se analizaron mediante el programa estadístico SAS ${ }^{\otimes}$ versión 9.4. (SAS, 2004); se realizó: test de normalidad, test de homogeneidad de varianza y análisis de Varianza (ANAVA), y prueba de comparación de medias (Tukey) con un nivel de significancia del 5\%.

\section{Resultados y Discusión}

\section{A. Variables de Cosecha}

A excepción del IM todas las variables de cosecha presentaron diferencias estadísticas $(\alpha=0,05)$ entre variedades. En el caso del IM todas las variedades presentaron valores de 0,95 a 1, esto indica que estas se encontraban en condiciones óptimas para cosecha (Ramírez et al., 2014a) y que para las condiciones que presenta el municipio de Barbosa, todos los materiales alcanzaron al mismo tiempo su madurez fisiológica.

La ALPQ a los 17 meses después de siembra fue en promedio de $2,82 \mathrm{~m}$, siendo la variedad CC 00-3257 la de mayor altura $(3,09 \mathrm{~m})$ clasificándose como tallos de mediana longitud $(2,50-3.50 \mathrm{~m})$ de acuerdo con Ramírez et al., (2014a) presentando diferencias estadísticas con la variedad CC 99-2461 que alcanzó 2,76 m de altura promedio (Tabla 1). Los datos encontrados son similares a los reportados por Ramírez-Durán et al. (2014b) en evaluaciones realizadas a variedades de caña de azúcar para panela en el departamento de Santander.

Para el DIATA se obtuvo un promedio de $2,84 \mathrm{~cm}$, y diferencias estadisticas $(\alpha=0,05)$ para las variedades CC $10-450$ y CC $11-600$ que presentaron los valores medios más bajos para DIATA (Tabla 1); el DIATA posee un efecto directo sobre el TCH, por lo que para obtener una alta producción de caña se requiere que haya altas poblaciones de tallos con diámetro superior a 2,5 $\mathrm{cm}$ (Viveros et al., 2015). Aunque el diámetro del entrenudo es una cualidad intrínseca de cada cultivar, determinada por sus características botánicas (Rodríguez-Seijo et al., 2020) esta variable también se ve afectada por el estado nutricional de la planta y su estado hídrico. La variedad CC 11-600 mostró uno de los valores inferiores para esta variable, sin embargo, uno de los valores más altos de TCH. Estos resultados son similares a los reportados por Ramírez-Durán et al. (2014b) con variedades de la serie 93.

Diatraea spp es considerado la plaga más nociva para las plantaciones de caña de azúcar, dado que su daño está relacionado directamente con efectos en la calidad del jugo que se traducen en disminución de rendimientos (Zúñiga-Oviedo \& Soto-Giraldo, 2018). Se observó que las variedades CC 00-3257, CC 11-600, CC 91-1606 y CC 99-2461 mostraron niveles bajos de intensidad de infestación y diferencias estadisticas con las demás variedades evaluadas 
TABLA I. Evaluación agronómica de variables de interés al momento de la cosecha para seis variedades de caña de azúcar (Saccharum officinarum L.), bajo condiciones del municipio de Barbosa, Santander (Colombia).

\begin{tabular}{|l|c|c|c|c|c|}
\hline Variedad & ALPQ $(\mathbf{m})$ & DIATA $(\mathbf{c m})$ & IM $(* *)$ & I.I & TCH \\
\hline CC 00-3257 & $3,09 \pm 0,143 \mathrm{a}$ & $3,18 \pm 0,227 \mathrm{a}$ & $0,98 \pm 0,016 \mathrm{a}$ & $0,09 \pm 0,011 \mathrm{~b}$ & $144,15 \pm 2,903 \mathrm{a}$ \\
\hline CC 00-3771 & $3 \pm 0,232 \mathrm{ab}$ & $3,44 \pm 0,07 \mathrm{a}$ & $1,03 \pm 0,034 \mathrm{a}$ & $0,17 \pm 0,032 \mathrm{a}$ & $95,2 \pm 7,147 \mathrm{~b}$ \\
\hline CC $10-450$ & $2,8 \pm 0,208 \mathrm{ab}$ & $1,98 \pm 0,009 \mathrm{~b}$ & $0,96 \pm 0,018 \mathrm{a}$ & $0,17 \pm 0,014 \mathrm{a}$ & $117,65 \pm 5,296 \mathrm{~b}$ \\
\hline CC $11-600$ & $2,78 \pm 0,045 \mathrm{ab}$ & $1,99 \pm 0,008 \mathrm{~b}$ & $0,99 \pm 0,018 \mathrm{a}$ & $0,06 \pm 0,012 \mathrm{~b}$ & $146,38 \pm 3,912 \mathrm{a}$ \\
\hline CC $91-1606$ & $2,48 \pm 0,547 \mathrm{~b}$ & $3,31 \pm 0,102 \mathrm{a}$ & $0,98 \pm 0,02 \mathrm{a}$ & $0,09 \pm 0,015 \mathrm{~b}$ & $103,42 \pm 4,144 \mathrm{~b}$ \\
\hline CC $99-2461$ & $2,76 \pm 0,125 \mathrm{ab}$ & $3,17 \pm 0,069 \mathrm{a}$ & $0,99 \pm 0,003 \mathrm{a}$ & $0,06 \pm 0,012 \mathrm{~b}$ & $106,72 \pm 5,711 \mathrm{~b}$ \\
\hline
\end{tabular}

Los valores corresponden con la media \pm el error estándar $(n=4)$; *Valores en la misma columna con diferente letra presentan diferencias significativas de acuerdo con la prueba múltiple de medias de Tukey $(a=0,05){ }^{* *}$ Adimensional; ALPQ: Altura de la planta a punto de quiebre; DIATA: Diámetro de tallo; IM: Índice de madurez; l.l intensidad de infestación por Diatraea; TCH: Toneladas de caña por hectárea.

(Tabla 1); el control químico de la plaga dado el habitat críptico que presentan en su estado larval es poco eficaz (López-Llano \& Soto-Giraldo, 2016), por lo que variedades con algun grado de resistencia al ataque de la plaga se convierten en una excelente alternativa de manejo y de renovación en variedades con alta susceptibilidad y bajos rendimientos como consecuencia del ataque de este insecto plaga.

En cuanto a la variable TCH, las variedades con mayor producción fueron CC 00-3257 y CC 11-600 con valores de 144,15 y 146,38 respectivamente, mostrando diferencias estadísticas con las demás variedades evaluadas (Tabla 1); estos valores son superiores al promedio reportado para la subregión de la Hoya del río Suárez donde se encuentra ubicado el municipio de Barbosa y cuyos rendimientos son de 100 TCH (González et al., 2018), de igual manera, el rendimiento en caña de estos materiales superan a lo obtenido por RD 75-11, variedad de mayor dominio en el área de estudio. De acuerdo con lo reportado por Viveros (2019), RD 75-11 alcanzó un rendimiento de 141,87 $\mathrm{TCH}$ en pruebas de evaluación agronómica realizado en contraste con otro grupo de variedades.

\section{B. Análisis de jugos y panela en laboratorio.}

Las variables conversión a panela (CP) y cachaza (Chz) no presentaron diferencias estadísticas entre variedades; la CP presentó valores entre el 10-12 \% estos datos son similares a los referidos por González et al. (2018) quienes indicaron que para la región de la Hoya del Río Suárez se presentaba en general una CP del $10 \%$. La Chz mostró valores inferiores (1\%-2,5\%) a los reportados por Durán (2010) quien indica que estos oscilan entre 3,0-4,5\% del total de la caña molida.

Los sólidos solubles totales medidos en jugo estuvieron entre 18,77 ${ }^{\circ}$ Brix 22,77 Brix presentando diferencias significativas entre variedades; las variedades CC 00-3771 y CC 10-450 registraron los menores valores para esta variable con $18,77^{\circ}$ Brix y $18,9^{\circ}$ Brix respectivamente (Figura 1 ), estos valores 
se encuentran dentro de los óptimos (17-22 'Brix) para obtener panela de buenas características comerciales (Ramírez Durán et al., 2014); resultados similares fueron encontrados por Lopez \& Tamayo (2017) y Ramírez-Durán et al. (2014b) en evaluaciones de variedades CC en Colombia.

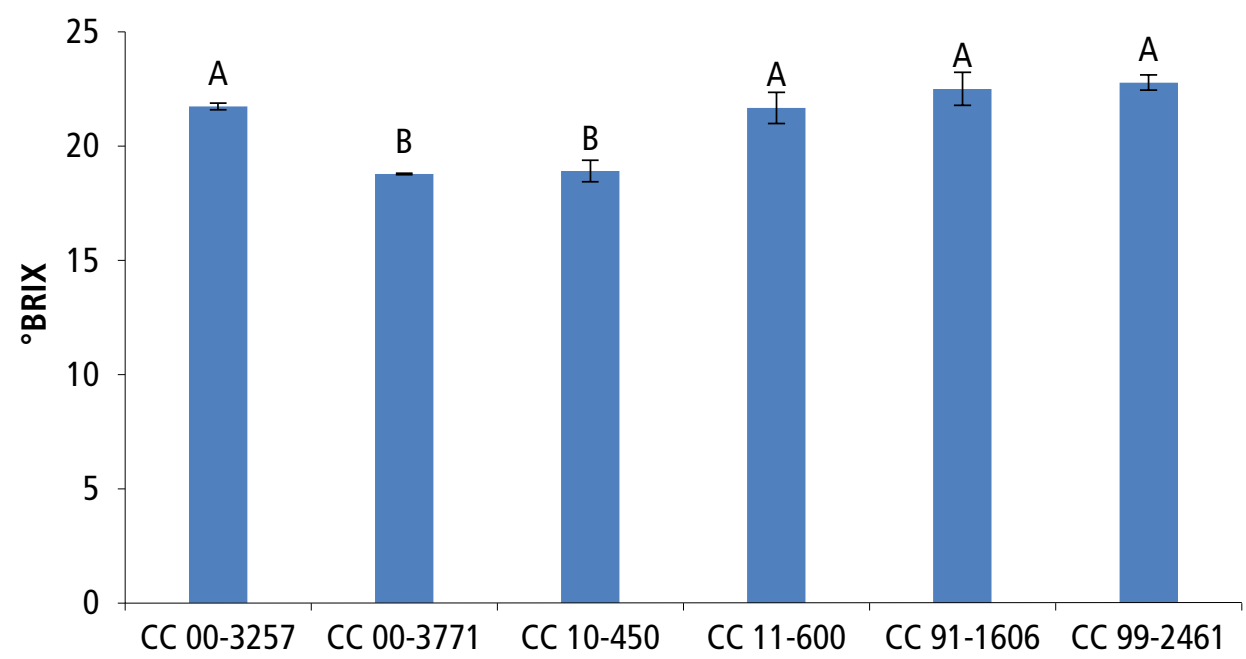

FIGURA 1. Solidos solubles totales ('Brix) en jugo de caña de azúcar de variedades cosechadas en Barbosa (Santander, Colombia). Medias con letra común no presentan diferencias estadísticas $(a=0,05)$. Barras verticales representan el error estándar $n=3$.

La estimación de toneladas de panela por hectárea (TPH) mostró diferencias estadísticas $(\alpha=0,05)$, las variedades CC 00-3257 y CC 11-600 mostraron los valores más altos con 16,93 y 16,33 TPH respectivamente, y diferencias con las demás variedades evaluadas (Figura 2). Con excepción de la variedad CC 00-3771, la producción de TPH por las variedades evaluadas fue superior a lo reportado para la región de la hoya del río Suárez (10 TPH) (González et al.,2018) y al rendimiento reportado por Rodríguez et al., 2019 de 10,57 t ha-1 para el departamento de Santander.

La calidad de la panela en Colombia está reglamentada por la resolución 779:2006 y NTC 1311:2009 cuyo objeto establece "los requisitos y los ensayos que debe cumplir la panela destinada para el consumo humano" (ICONTEC, 2009); las variables de calidad de panela no presentaron diferencias estadísticas $(\alpha=0,05)$ (Tabla 2). De igual manera es importante mencionar que para el presente estudio el contenido de azúcares no reductores (sacarosa), azúcares reductores y humedad obtenidos con los diferentes materiales evaluados estuvieron dentro de los rangos reportados como permitidos de acuerdo con lo estipulado en las diferentes reglamentaciones de calidad de panela para Colombia. 


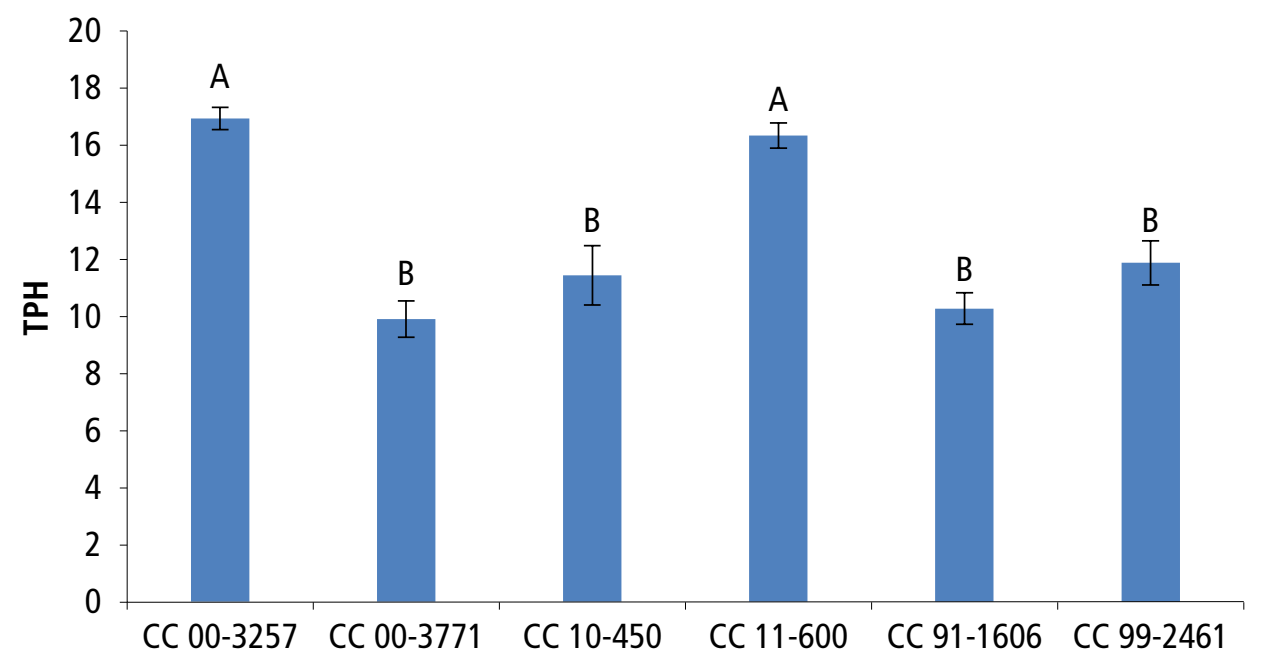

FIGURA 2. Toneladas de panela por hectárea (TPH) en evaluación de variedades de caña de azúcar para panela en Barbosa (Santander, Colombia). Medias con letra común no son significativamente diferentes $(a=0,05)$. Barras verticales representan el error estándar $n=3$.

Los sólidos solubles totales (SST) oscilaron entre 91,57 y $92,31{ }^{\circ} B$ rix, y concuerdan con lo descrito por Ramírez-Durán et al. (2014b) quienes afirman que los rangos normales para esta variable se encuentran entre $89-95^{\circ}$ Brix y encontraron una concentración promedio de $94,4^{\circ}$ Brix en evaluaciones de variedades CC de la series 93 a 99.

Los azucares reductores variaron entre $8,67 \%$ y $13,15 \%$ con un promedio de $10,93 \%$ estos valores estan acordes con lo reglamentado por ICONTEC (2009) y el Ministerio de la protección Social (2006) que establece que la panela en bloque debe contener como minimo un $5 \%$ de Azucares reductores. Sin embargo, en sus trabajos Durán-Castro (2010) y Volverás-Mambuscay et al. (2020) afirman que altos en esta variable podrían generar problemas de calidad en cuanto a consistencia y vida util.

TABLA II. Variables de calidad de panela obtenida para seis variedades de caña de azúcar (Saccharum officinarum L.) bajo condiciones de Barbosa, Santander (Colombia).

\begin{tabular}{|l|c|c|c|c|c|}
\hline Variedad & SST & Reductores & Humedad & Pureza & Sacarosa \\
\hline CC 00-3257 & $91,73 \pm 0,44$ & $8,67 \pm 0,63$ & $8,40 \pm 0,17$ & $86,13 \pm 1,18$ & $78,99 \pm 0,71$ \\
\hline CC 00-3771 & $92,22 \pm 0,88$ & $11,42 \pm 1,57$ & $9,11 \pm 0,29$ & $81,74 \pm 2,8$ & $75,34 \pm 1,98$ \\
\hline CC 10-450 & $91,57 \pm 0,16$ & $12,33 \pm 0,93$ & $9,91 \pm 0,53$ & $80,94 \pm 1,6$ & $74,11 \pm 1,33$ \\
\hline CC 11-600 & $91,96 \pm 0,29$ & $9,58 \pm 1,89$ & $9,22 \pm 0,62$ & $83,96 \pm 3,01$ & $77,19 \pm 2,55$ \\
\hline CC 91-1606 & $92,31 \pm 0,23$ & $10,45 \pm 0,82$ & $8,36 \pm 0,06$ & $82,75 \pm 0,73$ & $76,38 \pm 0,5$ \\
\hline CC 99-2461 & $91,97 \pm 0,80$ & $13,15 \pm 1,93$ & $8,80 \pm 0,34$ & $77,79 \pm 3,37$ & $71,58 \pm 3,49$ \\
\hline
\end{tabular}

Los valores corresponden con la media \pm el error estándar ( $n=3)$, SST ('Brix), las demás variables se expresan en porcentaje (\%). SST: Sólidos solubles totales 
La humedad promedio fue de $8,97 \%$ valor que se encuentra dentro de lo reglamentado por ICONTEC, (2009); la pureza y la sacarosa mostraron valores promedio de $82,22 \%$ y $75,6 \%$ valor normal para lo dispuesto por el Ministerio de la protección Social, (2006) e ICONTEC, (2009); resultados similares fueron reportados por Barona-Rodriguez et al. (2020), Lopez \& Tamayo (2017) y Ramírez Durán et al. (2014b) en evaluaciones de variedades de caña de azúcar para panela en Colombia.

\section{Conclusiones}

Las variedades CC 00-3257 y CC 11-600 mostraron buen comportamiento agronómico, fitosanitario e industrial, superando los rendimientos reportados para la región de la Hoya del Rio Suarez. En cuanto a la calidad de la panela obtenida, para las variables analizadas, todas las variedades cumplen con los requerimientos establecidos en la NTC 1311:2009 y la resolución 779:2006 del ministerio de salud y protección social.

Se recomienda la evaluación de las variedades CC 11-600 y CC 00-3257 en pruebas de evaluación agronómica en otras zonas paneleras y comparadas con un material testigo predominante.

\section{Agradecimientos}

Los autores agradecen a la Corporación Colombiana de Investigación Agropecuaria - Agrosavia el desarrollo de esta investigación enmarcada en el proyecto "Selección de nuevas variedades de caña de azúcar para panela, con alta productividad agroindustrial, amplia adaptabilidad, tolerantes a los factores bióticos y abióticos de las zonas paneleras de Colombia"; al Centro de Investigación de la caña de Azúcar de Colombia - Cenicaña por el aporte de los cultivares que pueden ser evaluados con enfoque de producción de panela; al Ministerio de Agricultura y Desarrollo Rural financiador de la investigación.

\section{Referencias}

Barona-Rodriguez, A. F., Insuasty-Burbano, O., Viveros-Valens, C. A., Ángel-Sánchez, J. C., \& Ramírez-Durán, J. (2020). Evaluación de cultivares de caña de azúcar (Saccharum spp.) para producción de panela en el departamento de Boyacá, Colombia. Revista U.D.C.A Actualidad \& divulgación Científica, 23(2), 1-9. http://doi. org/10.31910/rudca.v23.n2.2020.1298

Cenicaña. (2005). Evaluación del daño causado por Diatraea spp. en caña de azúcar y su manejo en el valle del río. Cali: Serie divulgativa Numero 9. Obtenido de https://www.cenicana.org/pdf_privado/serie_divulgativa/sd_09/sd_09.pdf

Delgado-Mora, I., Nuñez-Jaramillo, D., Jorge-Suarez, H., Guillen-Sosa, S., Díaz-Mujica, F., Gómez-Pérez, J., . . . Montes de Oca, J. (2016). Evaluación de cultivares 
de caña de azúcar de madurez temprana, para el inicio de la zafra azucarera en suelos sialitizados no cálcicos. Centro Agricola, 43(2), 5-13, http://scielo.sld. $\mathrm{cu} / \mathrm{pdf} / \mathrm{cag} / \mathrm{v} 43 \mathrm{n} 2 / \mathrm{cag} 01216 . \mathrm{pdf}$

Duarte-Álvarez, O., González-Villalba, J. (2019) Guía técnica cultivo de caña de azúcar San Lorenzo, Paraguay: FCA, UNA, 2019. Obtenido de: https://www.jica. go.jp/paraguay/espanol/office/others/c8h0vm0000ad5gke-att/gt_01.pdf

Durán Castro, N. (2010). Reíngenieria Panelera. Tunja, Colombia: Buhos Editores. FAO. (1994). DEFINITION AND CLASSIFICATION OF COMMODITIES; 3. SUGAR CROPS AND SWEETENERS AND DERIVED PRODUCTS. Recuperado el 18 de 08 de 2021, de Organización de las naciones unidas para la alimentación y la agricultura: http://www.fao.org/es/faodef/faodefe.htm

Fedepanela. (05 de 02 de 2021). Federación Nacional de productores de panela. Obtenido de https://fedepanela.org.co/gremio/

Flórez-Martínez, D. H., Contreras-Pedraza, C. A., \& Rodríguez, J. (2021). A systematic analysis of non-centrifugal sugar cane processing: Research and new trends. Trends in Food Science \& Technology, 107, 415-428. https://doi.org/10.1016/j. tifs.2020.11.011

Garcia, M. J., Narváez, P. C., Heredia, F. J., Orjuela, A., \& Osorio, C. (2017). Physicochemical and sensory (aroma and colour) characterisation of a non-centrifugal cane sugar ("panela") beverage. Food Chemistry, 228, 7-13. https://doi. org/10.1016/j.foodchem.2017.01.134

Gimenes-Catelan, M., Marques-Júnior, J., Silva-Siqueira, D., Pimenta-Gomez, R., \& Bahia, A. (2022). Sugarcane yield and quality using soil magnetic susceptibility. Scientia Agricola, 79(4), 11. https://doi.org/10.1590/1678-992X-2020-0329

Gómez-Merino, F., Trejo-Tellez, L. I., Salazar-Ortiz, J., Perez-Sato, J. A., H.E, S.-H., Bello-Bello, J. J., \& Aguilar-Rivera, N. (2017). La diversificación de la industria azucarera como estrategía para México. Agro-Productividad, 10(11), 7-12. https://revista-agroproductividad.org/index.php/agroproductividad/article/ view/73/68

González, F., Cabezas, M., Ramírez, M., \& Ramírez, J. (2018). Curvas de absorción de macronutrientes en tres variedades de caña de azúcar (Saccharum officinarum L.) para panela, en la hoya del río Suaréz. Revista U.D.C.A Actualidad \& Divulgación Cientifica, 21(2), 395-404. https://doi.org/10.31910/rudca.v21. $\mathrm{n} 2.2018 .995$

ICONTEC. (2009). NTC 1311 (Tercera actualización ed.). Bogotá: Instituto Colombiano de Normas Técnicas y Certificación (ICONTEC).

Jaffé, W. R. (2015). Nutritional and functional components of non centrifugal cane sugar: A compilation of the data from the ana. Journal of food composition analysis, 43, 194-202. https://doi.org/10.1016/j.jfca.2015.06.007

Lopez, J. G., \& Tamayo, Á. (2017). Agroindustrial performance of sugarcane varieties for panela in Antioquia, Colombia. Revista Facultad Nacional de Agronomia, 70(3), 8303-8310. https://doi.org/10.15446/rfna.v70n3.66329

López-Llano, R. A., \& Soto-Giraldo, A. (2016). Aislamiento de nematodos entomopatógenos nativos en cultivos de caña panelera y pruebas de patogenicidad sobre Diatraea saccharalis (Lepidoptera: Crambidae). Boletín Científico centro de museos museo de historia natural, 20(2), 114-123. https://revistasojs.ucaldas. edu.co/index.php/boletincientifico/article/view/3799/3509 
Mendoza-Batista, Y., Cruz-Sarmiento, R., Vaillant-Cáceres, Y., Luis-Martínez, O., \& Céspedes-Argota, M. (2019). Comportamiento de los cultivares de caña de azúcar C97-445 y C95-416 en localidades de la provincia Holguín. Centro Agricola, 46(1), 49-53. http://scielo.sld.cu/pdf/cag/v46n1/0253-5785-cag-46-01-49.pdf

Ministerio de la protección Social. (2006). Resolución Número 000779 de 2006. Bogotá: Diario Oficial 46223 de 2006. Obtenido de https://www.minsalud.gov. co/sites/rid/Lists/BibliotecaDigital/RIDE/DE/DIJ/Resolucion-779-de-2006.pdf

Murcia-Pardo, M., \& Ramírez-Durán, J. (2017). Reconversión del sistema regional de producción de caña para la agroindustria panelera en Boyacá y Santander. Ciencia y Tecnología Agropecuaria, 18(1), 75-87. http://dx.doi.org/10.21930/ rcta.vol18_num1_art:559

Ramírez Durán, J., Insuasty Burbano, O., \& Murcia Pardo, M. L. (2014a). Variedades de caña de azúcar empleadas para la agroindustria panelera de Colombia. Barbosa, Colombia: Corpoica. https://repository.agrosavia.co/ handle/20.500.12324/12621

Ramírez Durán, J., Insuasty Burbano, O., \& Viveros Valens, C. A. (2014b). Comportamiento agroindustrial de diez variedades de caña de azúcar para producción de panela en Santander, Colombia. Ciencia y Tecnología Agropecuaria, 15(2), 183-195. https://doi.org/10.21930/rcta.vol15_num2_art:358

Ramírez-Durán, J., Cañar-Serna, D. Y., Deantonio-Florido, L. Y., \& Hernández-Nopsa, J. F. (2019). Manual técnico para la producció de plántulas de caña de azúcar para panela a partir de yemas individuales bajo las condiciones agroecológicas del municipio de Barbosa (Santander). Mosquera, Colombia: Agrosavia. https:// repository.agrosavia.co/handle/20.500.12324/35065

Rodríguez, G. A., Polo, S. M., Ángel, M., Buitrago, A. M. (2019). La agroindustria panelera impulsando el desarrollo rural en Colombia. Bogotá. 55p. Roffaprint Editores S.A.S.

Rodríguez-Seijo, I., Martínez-Campos, P., Alvárez-Hernández, U., Cruz-Limonte, A., \& Mora-Pérez, E. (2020). Efectos del surco de base ancha sobre el crecimiento y el rendimiento agrícola de la caña de azúcar (Saccharum spp.). Centro Agrícola, 47(2), 66-74. http://scielo.sld.cu/pdf/cag/v47n2/0253-5785-cag-47-02-66.pdf

Suárez, H., González-Marrero, A., Menéndez-Sierra, A., \& Vera-Méndez, A. (2019). Selección de cultivares de caña de azúcar estables y productivos en la compañía azucarera La Estrella. Biotecnología Vegetal, 19(4), 307-315. http://scielo.sld. cu/pdf/bvg/v1 9n4/2074-8647-bvg-19-04-307.pdf

Velásquez, F., Espitia, J., Mendieta, O., Escobar, S., \& Rodriguez, J. (2019). Non-centrifugal cane sugar processing: A review on recent advances and the influence of process variables on qualities attributes of final products. Journal of Food Engineering, 255, 32-40. https://doi.org/10.1016/j.jfoodeng.2019.03.009

Vera-Gutiérrez, T., Garcia-Muñoz, M. C., Otálvaro-Alvarez, A. M., \& Mendieta-Menjura, O. (Octubre de 2019). Effect of processing technology and sugarcane varieties on the quality properties of unrefined non-centrifugal sugar. Heliyon, 5(10). https://doi.org/10.1016/j.heliyon.2019.e02667

Viveros, C. A., Baena, D., Salazar, F., López, L. O., \& Victoria, J. I. (2015). Caracteristicas de la caña de azúcar asociadas con toneladas de caña por hectárea y sacarosa (\%caña). Acta Agronómica, 64(3), 268-272. http://dx.doi.org/10.15446/ acag.v64n3.44494 
Viveros-Valens, C.A. (2019). Variedades de caña de azúcar Cenicaña Colombia (CC) evaluadas en las zonas paneleras de Colombia. Cali: Fondo de Fomento Panelero (Fedepanela). $92 \mathrm{p}$.

Volverás-Mambuscay, B., González-Chavarro, C. F., Huertas, B., Kopp-Sanabria, E., \& Ramírez-Durán, J. (2020). Efecto del fertilizante orgánico y mineral en rendimiento de caña panelera en Nariño, Colombia. Agronomía Mesoamericana, 31(3), 547-565. https://doi.org/10.15517/am.v31i3.37334

Zúñiga-Oviedo, M. A., \& Soto-Giraldo, A. (2018). CONTROL MICROBIOLÓGICO DE Diatraea saccharalis Fabricius (LEPIDOPTERA: CRAMBIDAE) EN CAÑA PANELERA A NIVEL DE CAMPO. Boletín Cientifíco centro de museos museo de historia natural, 22(2), 33-41. https://revistasojs.ucaldas.edu.co/index.php/boletincientifico/ article/view/2684/2482 
